\title{
Silencing of Rac1 modifies lung cancer cell migration, invasion and actin cytoskeleton rearrangements and enhances chemosensitivity to antitumor drugs
}

\author{
QING-YONG CHEN $^{1 *}$, LI-QUN XU ${ }^{2 *}$, DE-MIN JIAO ${ }^{1}$, QING-HUA YAO $^{3}$, YAN-YI WANG $^{4}$, \\ HUI-ZHEN HU ${ }^{1}$, YU-QUAN WU ${ }^{1}$, JIA SONG $^{1}$, JIE YAN $^{1}$ and LI-JUN WU ${ }^{1}$
}

\author{
Departments of ${ }^{1}$ Respiratory Disease and ${ }^{2}$ Oncology Disease, The 117th Hospital of PLA, Hang Zhou 310013; \\ ${ }^{3}$ Department of Respiratory Disease, The First Affiliated Hospital, Zhejiang Chinese Medical University, \\ Hang Zhou 310053; ${ }^{4}$ College of Life Science, Guizhou University, Guiyang 550025, P.R. China
}

Received May 27, 2011; Accepted June 24, 2011

DOI: 10.3892/ijmm.2011.775

\begin{abstract}
Rac1, an intracellular signal transducer, regulates a variety of cell functions, including the organization of the cytoskeleton, cell migration, and invasion. Overexpression of Rac1 has been reported in several human cancers. However, the underlying mechanisms are not well understood. In the present study, we evaluated the possibility of Rac1 as an appropriate molecular target for cancer gene therapy. The expression of Rac1 in 150 primary non-small cell lung cancer tissues (NSCLC) and 30 normal paraneoplastic lung tissues was determined by immunohistochemical staining, and the correlation of Rac1 overexpression with clinicopathological factors was evaluated. Overexpression of Rac1 was detected in 94 of 150 lung cancer specimens, the incidence rate being higher than that in normal lung tissue specimens. In addition, overexpression of Rac1 was also associated with poor differentiation, high TNM stage, and lymph node metastasis in NSCLC patients. Moreover, RNAi-mediated suppression of Racl expression reduced lamellipodia formation, migration and invasion potential of a lung cancer cell carcinoma cell line, 801D. Down-regulation of Rac1 expression also reduced the expression of Pak1. NSC23766, an inhibitor of Rac1 activity, could also inhibit lung cancer cell migration, invasion and induce rearrangements of the actin cytoskeleton. Furthermore, the suppression of Racl expression also sensitized cells to
\end{abstract}

Correspondence to: Dr Qing-Yong Chen, Department of Respiratory Disease, The 117th Hospital of PLA, Hang Zhou 310013, P.R. China E-mail: cqyong117@163.com

Dr Li-Qun Xu, Department of Oncology Disease, The 117th Hospital of PLA, Hang Zhou 310013, P.R. China

E-mail: 5190858@163.com

${ }^{*}$ Contributed equally

Key words: Rac1, RNA interference, actin cytoskeleton, migration and invasion, lung cancer cells, chemosensitivity antitumor drugs. These results indicate that the overexpression of Racl is tightly associated with an aggressive phenotype of lung cancer cells. Therefore, we proposed that Racl could be a potential molecular target of gene therapy by RNAi-targeting in lung cancer cells.

\section{Introduction}

Accumulating evidence indicates that small GTPase-dependent cell signaling is important for malignant migration and the motility process (1). The Rho family GTPases belong to the Ras superfamily of small GTPases that serve as molecular switches. Activation of Rho family GTPases regulates a wide range of cellular functions. Racl is an important GTPase that has been implicated in many cellular processes, such as cytoskeleton rearrangement, cell adhesion, and transcriptional activation, and is believed to be involved in cancer cell migration, invasion, and metastasis (2,3). A number of investigations indicate that Rac1 plays an important role in malignant transformation. Rac1 overexpression has been found in breast carcinoma, gastric carcinoma, oral squamous cell carcinoma (SCC), non-small cell lung carcinoma, and testicular germ cell tumors. Racl overexpression has also been considered as an independent predictor of adverse outcome of these carcinomas $(4,5)$. Consistent with these reports, suppression of Rac1 protein expression and disruption of its function significantly reduced lung metastasis in a mouse colorectal adenocarcinoma model (6). Furthermore, Racl silencing by small hairpin RNA could reverse the metastatic behavior of human glioblastoma cells and fibrosarcoma HT1080 cells $(7,8)$. Taken together, the observed effects of Racl overexpression and silencing on the cell malignant transformation indicate a role for Rac1 in regulating tumor metastasis and progression.

In addition, Racl can interact with various specific effectors to coordinate the activation of a multitude of signaling cascades that influence diverse physiological outcomes. The Pak (p21-activated kinase) serine/threonine kinases have recently been found to be key regulators of cytoskeletal remolding, cell motility, and cell proliferation, with a role in both carcinogenesis and cellular invasion (9). It has been reported that Pak1, the best 
characterized member of this family, shows increased expression and activity in human cancers $(9,10)$. Multiple signaling pathways converge to promote activation of Pak1 through both small GTPases and several of the tyrosine kinases. In turn, activated Pak1 regulates diverse cellular functions. Pak1 binds to Rac1 in a GTP-dependent manner, after which activated Pak1 regulates cellular functions such as cytoskeletal dynamics, cell adhesion, and transcription (9).

However, the mechanisms of Rac1-mediated tumor development still require further elucidation. In the present study, we showed that overexpression of Racl was widespread in primary lung cancer patients. Knocking down of Rac1 expression by short hairpin RNA (shRNA) against Rac1 suppressed lung cancer cell migration, invasion and induced rearrangements of the actin cytoskeleton in lung cancer cells. Racl suppressed lung cancer cell migration through Pak1-dependent cell signaling pathways. All these results suggested that Rac1 may be an adjuvant gene therapy strategy to chemotherapy.

\section{Materials and methods}

Reagents and cell lines. Rac1 antibodies were purchased from Santa Cruz Biotechology (Santa Cruz, CA). Phosphorylated Pak1 and total Pak1 antibodies, MTT, IPA3 and TRITCphalloidin were purchased from Sigma. Human lung cancer cell lines (95D, 801D, PG, A549 and 95C) and human bronchial epithelial cells (BEAS-2B) (a gift from Dr Guohua Lu, Zhejiang University) were cultured in RPMI-1640 medium at $37^{\circ} \mathrm{C}$ in $5 \% \mathrm{CO}_{2}$. Rac1-shRNA (Racl target sequence 5'-GCGATCCGAGCAGTCCTCT-3') plasmid (pGPU6/ Neo-Rac1) and empty plasmid vector (pGPU6/GFP/Neo-control) were obtained from Zhe Jiang Chinese Medical University. Elemene was obtained from Huali Jin Gang Pharmaceuticals Ltd., Inc., Dalian, P.R. China. Nedaplatin was obtained from the 117th Hospital of PLA (Hang Zhou, China).

Patients and immunohistochemistry. This study was approved by the Institutional Review Board of the 117th Hospital of PLA. Surgically-resected specimens from 150 patients (100 males and 50 females) with lung cancer were examined. The mean age of the patients was 47.5 years (range, 22-77 years). This patient population represented a randomly selected subgroup from a clinical series including all patients who underwent surgical resection between June 2002 and December 2004 in the Thoracic Surgical Department of the 117th Hospital of PLA. The diagnosis of lung cancer, histological grading and pathological staging were re-evaluated and/or confirmed by two independent pathologists. Follow-up information was obtained from review of the patients' medical records. None of the patients had received radiotherapy or chemotherapy before surgical resection and all the patients were treated with routine chemotherapy after the operation. The histological diagnosis and grade of differentiation were evaluated using hematoxylin and eosin-stained sections according to the World Health Orgnanization (WHO) guidelines of classification (2004) (11). All 150 specimens were re-evaluated with respect to histologic subtype, differentiation, and tumor stage, following collection. SCC was identified in 55 of the cases, and adenocarcinoma in 95 of the cases (56 well-differentiated, 64 moderately differentiated, and 30 poorly differentiated). Lymph node metastases were identified in 80 of the 150 patients. The TNM staging system of the International Union Against Cancer (12) was used to classify specimens as stages I $(n=58)$, II $(n=57)$, and III ( $\mathrm{n}=35)$. Immunohistochemical staining and evaluation were described in previous studies (13).

Cell transfections. The shRNA expression plasmids were transfected into 801D cells using Lipofectamine 2000 (Invitrogen, USA) as described previously (14). Stable GFP-positive clones were obtained by flow sorter. The expanded cells were then used for subsequent studies. Racl expression was determined by Western blotting.

Migration and invasion assay. Wound healing experiment and Transwell insert (24-well insert; pore size, Corning, USA) assays were used to explore the effect of Racl on the migration and invasion, respectively, of $801 \mathrm{D}$ cells as previously described (14). Briefly, for the wound healing experiment, cells were grown to confluence wounded using a pipette tip and photographed at $0 \mathrm{~h}$ and subsequent time points. Cell migration was evaluated by measuring the width of the wound at the identical position. For the invasion assay, the lower chambers of matrigel-coated invasion plates were used. Cells $(50,000)$ were added to the upper chamber in serum-free media and invasion at $37^{\circ} \mathrm{C}$ towards $10 \%$ FBS-containing growth media was determined after $24 \mathrm{~h}$. Cells that invaded through the membrane were fixed, stained with crystal violet stain and counted with light microscopy. All experiments were carried out in triplicate.

Cell proliferation analysis. To analyze the effect of Rac1 on 801D cell proliferation activity, the MTT assay was performed. Briefly, cells were plated into 96-well plates at 5,000 cells/well, cultured in 10\% FBS RPMI-1640 for various durations and then MTT solution $(5 \mathrm{mg} / \mathrm{ml})$ was added to the medium. The formazan crystals that formed were dissolved, and absorption was measured at $490 \mathrm{~nm}$ with an automatic ELISA reader.

Confocal microscopy. Cells were washed thrice with PBS and fixed with $4 \%$ paraformaldehyde for $10 \mathrm{~min}$ at room temperature. Following three washes with PBS, cells were permeabilized for 5 min with $0.5 \%$ Triton X-100 dissolved in PBS. Cells were then incubated with rhodamine-conjugated phalloidin (Molecular Probes, USA) for $30 \mathrm{~min}$ to stain for F-actin. The cells were analyzed by laser confocal microscopy and photographed.

Western blot analysis. Cells were lysed in a lysis buffer $(50 \mathrm{mM}$ Tris-HCl, pH 7.8, 150 mM NaCl, 1\% NP-40, 0.1\% SDS, 1 mM phenylmethylsulfonyl fluoride) as previously described (15). Briefly, the lysates were resolved on 10\% SDS-PAGE gels, then transferred to PVDF membranes, blocked with 5\% non-fat milk, probed with primary and corresponding secondary antibodies and detected by an ECL reagent.

Drug-sensitivity assay. To assess the chemosensitvity to antitumor drug, the cells were seeded in triplicates on 96-well plates at 5,000 cells/well and incubated for $24 \mathrm{~h}$. The medium was then removed and replaced with fresh medium containing varying concentrations of drugs. After $24 \mathrm{~h}$, cells were treated 
Table I. Correlation of Rac1 expression with clinicopathological features.

\begin{tabular}{|c|c|c|c|c|c|}
\hline \multirow[b]{2}{*}{ Variable } & \multirow[b]{2}{*}{$\mathrm{n}$} & \multicolumn{2}{|c|}{ Rac1 expression } & \multirow[b]{2}{*}{$\chi^{2}$} & \multirow[b]{2}{*}{ P-value } \\
\hline & & Positive $(n=94)$ & Negative $(\mathrm{n}=56)$ & & \\
\hline \multicolumn{6}{|l|}{ Gender } \\
\hline Male & 100 & 62 & 38 & 1.16 & 0.28 \\
\hline Female & 50 & 32 & 28 & & \\
\hline \multicolumn{6}{|l|}{ Age } \\
\hline$\geq 50$ years & 70 & 44 & 26 & 0.002 & 0.96 \\
\hline$<50$ years & 80 & 50 & 30 & & \\
\hline \multicolumn{6}{|l|}{ Tumor size } \\
\hline$\geq 3 \mathrm{~cm}$ & 79 & 51 & 28 & 0.255 & 0.61 \\
\hline$<3 \mathrm{~cm}$ & 71 & 43 & 28 & & \\
\hline \multicolumn{6}{|l|}{ TNM stage } \\
\hline $\mathrm{I}+\mathrm{II}$ & 115 & 85 & 30 & 4.862 & 0.027 \\
\hline III & 35 & 19 & 16 & & \\
\hline \multicolumn{6}{|l|}{ Differentiation } \\
\hline Well/moderately & 120 & 68 & 52 & 9.233 & 0.002 \\
\hline Poorly & 30 & 26 & 4 & & \\
\hline \multicolumn{6}{|l|}{ Histological type } \\
\hline Squamous cell carcinoma & 55 & 36 & 29 & 0.512 & 0.47 \\
\hline Adenocarcinoma & 95 & 58 & 37 & & \\
\hline \multicolumn{6}{|l|}{ Lymph node metastases } \\
\hline Negative & 70 & 34 & 36 & 11.146 & 0.001 \\
\hline Positive & 80 & 60 & 20 & & \\
\hline
\end{tabular}

with MTT as described above. The inhibition ratio was calculated. The assay was repeated three times.

Statistical analysis. SPSS version 11.5 for Windows was used for all analyses. The $\chi^{2}$-test was used to examine possible correlations between the expression profiles of Racl, and their all-abnormal expression, as well as for the comparison with clinicopathological factors. The data were expressed as mean \pm SD and one-Way ANOVA test was used to determine the significance of the multiple comparisons. The KaplanMeier method was used to estimate the probability of patient survival, and the log-rank test was used to evaluate differences in survival between patient subgroups. Differences were considered significant when $\mathrm{P}<0.05$.

\section{Results}

Immunohistochemical analysis of Racl expression in lung cancer samples. To assess the role of Rac1 in lung cancer, we analyzed its expression pattern in 150 lung cancer cases and 30 normal paraneoplastic lung tissues $(>1.5 \mathrm{~cm}$ away from the tumor). Among these 30 cases of normal lung tissues, weak to no expression was observed (Fig. 1A); However, 94/150 (62.67\%) lung cancer samples were found to be Racl-positive, (Fig. 1B-E), suggesting that Rac1 was overexpressed in human lung cancer. Racl high expression was significantly associated

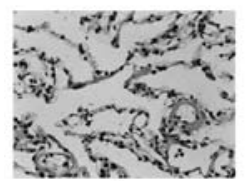

A

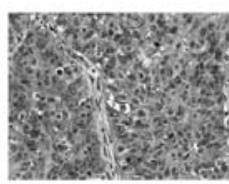

D

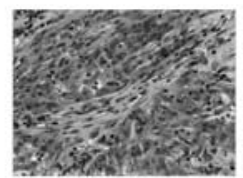

B

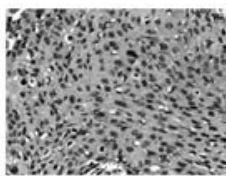

C

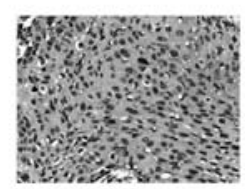

E

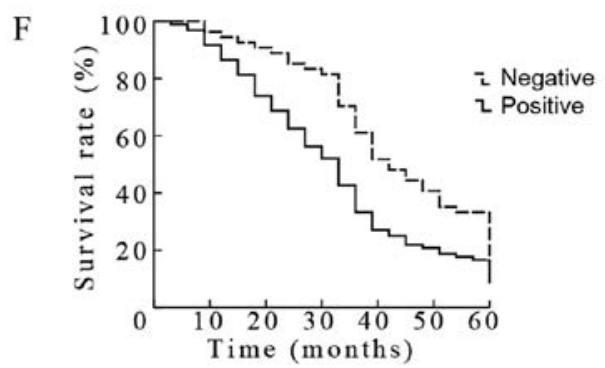

Figure 1. Expression of Rac1 in lung cancer tissues and its relation to overall survival. (A) Negative immunostaining for Rac1 in normal lung tissues. In contrast, Racl showed strong cytoplasmic staining in (B) lung adenocarcinoma and (C) lung squamous cell carcinoma (SCC), weak cytoplasmic staining in (D) lung adenocarcinoma and (E) lung SCC. (F) Kaplan-Meier survival curves were constructed, and the difference between the Rac1-positive and Rac1-negative groups was analyzed by log-rank test. Magnification, x200. 
A

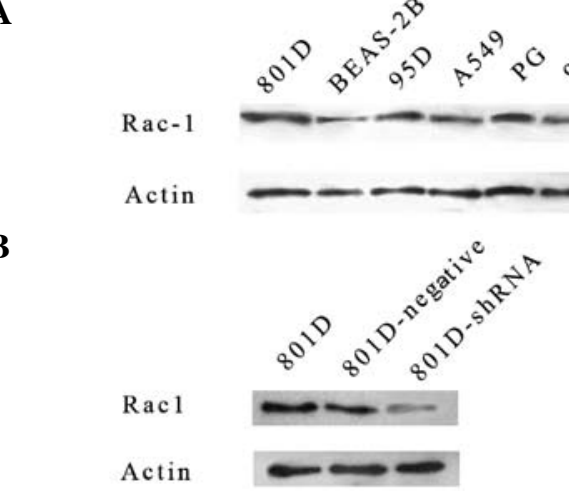

Figure 2. Expression of Rac1 in various lung cancer cells. Five human lung cancer cell lines (PG, 801D, 95D, A549 and 95C) were selected, Rac1 protein expression was detected by Western blotting (A). Deletion of Rac1 inhibits the expression of Rac1 in 801D cells (B). with lymph nodal metastasis, TMN staging and differentiation, but not correlated with other factors (such as age, gender, tumor size and histological type) (Table I). Additionally, Kaplan-Meier analysis revealed that the 5-year survival rate of patients with Rac1-negative expression was $32.14 \%$, which was significantly higher than that of patients with Rac1-positive expression $\left(17.02 \%, \chi^{2}=12.85, \mathrm{P}<0.005\right)(\mathrm{Fig} .1 \mathrm{~F})$. These results showed that Racl expression levels could serve as a prognosis and metastasis marker in lung cancer patients.

Expression of Racl in human lung cancer cell lines with different metastasis potentials. To clarify the functional relationship between Racl expression and the ability of tumor metastasis in human lung cancer cell lines PG, 801D, 95D, A549 and 95C, Rac1 protein levels were detected by Western blot analysis in these cell lines (Fig. 2A). Of the five human
A

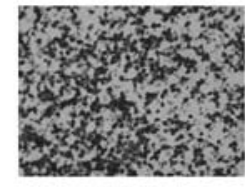

$801 \mathrm{D}$

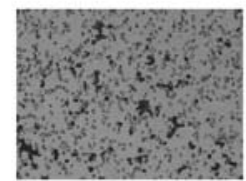

801 D-shRNA

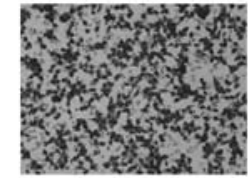

801 D-shRNAcontrol

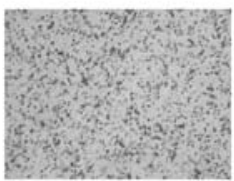

$801 \mathrm{D}-\mathrm{NSC} 23766$

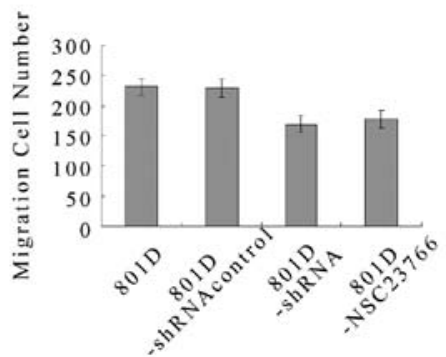

B

801D

$801 \mathrm{D}+$ control

$801 \mathrm{D}+$ shRNA

$801 \mathrm{D}+\mathrm{NSC} 23766$
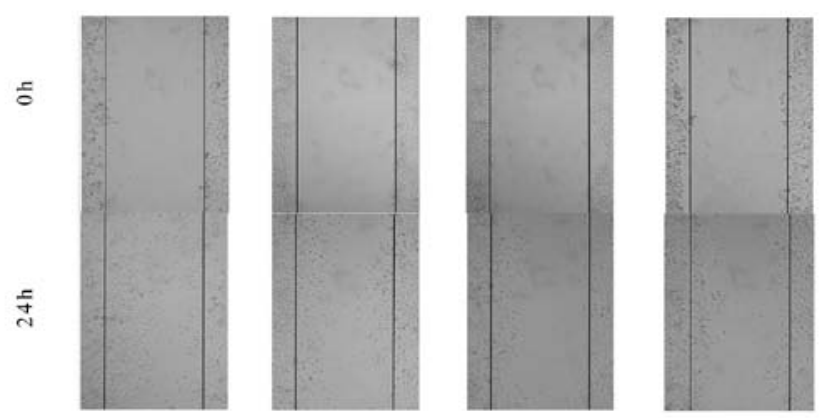

C

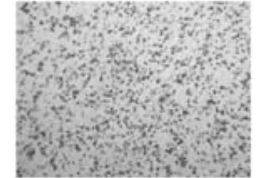

$801 \mathrm{D}$

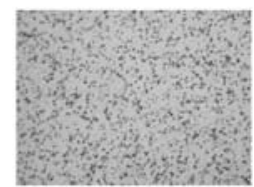

801 D-shRNA

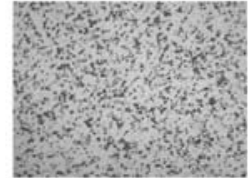

801D-shRNAcontrol

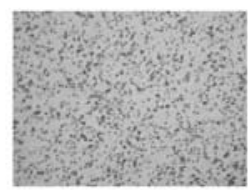

$801 \mathrm{D}-\mathrm{NSC} 23766$

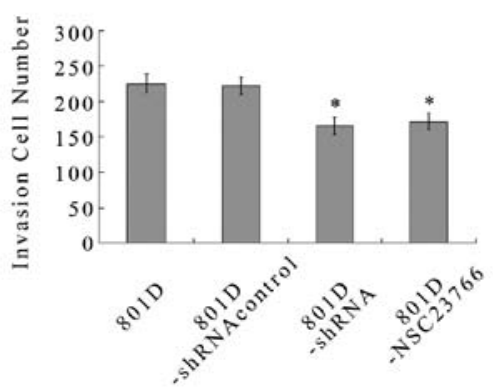

Figure 3. Transwell insert and wound healing assay showing that Rac1 regulates cell migration and invasion in vitro. After initial equilibrium, 801D cells un-transfected or transfected with Racl or negative control and treated with NSC23766 suspended in fresh medium without fetal bovine serum were added to the insert. (A) The migratory cell number of 801D transfected with Racl was significantly less than that of 801D cells transfected with negative control or un-transfected. (B) Confluent cell monolayers were wounded with a pipette tip. Wound closure was monitored by microscopy at the indicated times. (C) For the invasion assay, the inserts were coated with ECM and then repeated as for the migration assay. The invasive cell number of $801 \mathrm{D}$ transfected with Rac1 was also significantly lower than that of 801D cells transfected with negative control. Effects of NSC23766 treatment on the invasion of lung cancer cells is very similar to the effects of Rac1-shRNA. ${ }^{*} \mathrm{P}<0.05$ compared with control. 
lung cancer cell lines, Racl expression was the strongest in the highly metastatic cells 801D and PG, and the weakest in the lowly-metastatic 95C cells, suggesting that Racl expression is positively associated with metastatic potentials of human lung cancer cells. Interestingly, Racl expression in human bronchial epithelial cells (BEAS-2B) was very weak. In addition, expression of Racl protein decreased significantly in 801D cells with transfected Rac1-shRNA plasmid compared to the cells with shRNA-control, as shown by Western blot analysis (Fig. 2B). Together, these results suggested that Rac1 was overexpressed in highly metastatic lung cancer cells. Thus, 801D cells were selected to further investigate the effect of specific silencing of 801D on metastasis behaviors such as tumor cell invasion and cell motility.

Racl silencing suppresses $801 D$ cell migration and invasion. Increased cell motility and invasion of carcinoma cells are key steps in the metastatic cascade. We next examined the role of Rac1 in the cell motility ability of 801D cells. Wound healing experiment results showed that Rac1-shRNA expressing 801D cells exhibited reduced migration ability compared with the control shRNA cells or the cells with no transfectant (Fig. 3A). We then assayed the polarized migration of cells using the scratch-wound model. As shown in Fig. 3B, cells transfected with Rac1-shRNA closed the scratch-wounds more slowly than cells untreated or transfected with negative control. Consistent with the migration results, the silencing of Rac1 in Rac1shRNA expressing 801D cells significantly inhibited their invasion (Fig. 3C). These data demonstrated the importance of Racl in lung cancer cell migration and invasion.

NSC23766, a Racl inhibitor, inhibits lung cancer cell migration and invasion. This experiment was performed to determine whether inhibition of lung cancer cell migration is mediated through Racl expression. For this purpose, equal numbers of $801 \mathrm{D}$ cells were subjected to the cell migration assay after treatment with $100 \mu \mathrm{M}$ NSC23766, a Rac1 inhibitor for $24 \mathrm{~h}$. Treatment of the cells with NSC23766 resulted in a reduction in the cell migration and invasion capacity of lung cancer cells as compared with non-NSC23766-treated controls (Fig. 3). These data suggest that the inhibition of constitutive levels of Rac1 expression in the presence of NSC23766 resulted in inhibition of migration and invasion.

Effects of depletion of Racl on $801 D$ cell proliferation. To rule out the possibility that the stimulation of migration following Rac1 inhibition was due to stimulation of proliferation, we performed cell survival/proliferation assays in 801D cells under the same conditions as our migration assay. Results of these assays revealed no significant change in proliferation of shRNA-transfected cells (Fig. 4) as well as pharmacological Rac1 inhibitor-treated cells (data not shown), suggesting that the dramatic inhibition in cell migration caused by depletion of Racl is not due to a decrease in cell growth.

Racl is essential for rearrangements of the actin cytoskeleton in lung cancer cells. Because actin cytoskeletal reorganization is considered to be one of the most important functions of Rho GTPases, we stained untreated and NSC23766-treated 801D cells or shRNA-transfected cells for F-actin with TRITC-conjugated

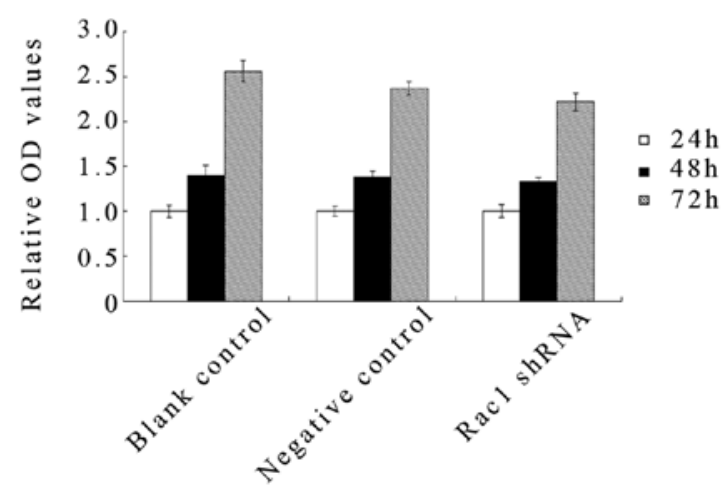

Figure 4. Effects of depletion of Rac1 on lung cancer cell proliferation. Cell numbers were determined after 24, 48 and $72 \mathrm{~h}$ as described in Materials and methods. Lack of significant inhibition of cell proliferation is observed in the graph.

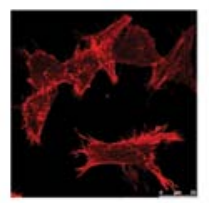

A

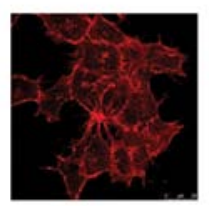

E

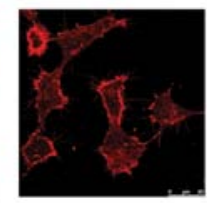

B

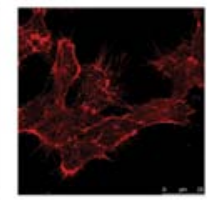

F

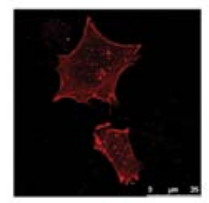

C

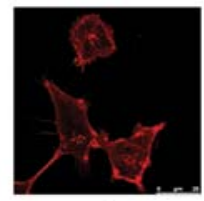

G

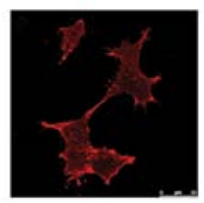

D

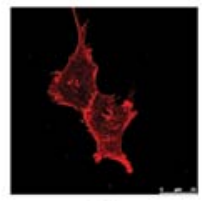

$\mathrm{H}$
Figure 5. Suppression of Rac1 expression by shRNA induces rearrangements of the actin cytoskeleton in lung cancer cells. 801D cells and 801D-negative control cells showed a cross-linked actin network (A and B). Deletion of Rac1 reduced the appearance of a cross-linked actin network in 801D-shRNA cells (C). Deletion of Rac1 inhibited the EGF-induced lamellipodia and pseudopodia formation (D), but this phenomenon was not shown in 801D cells and 801D-negative control cells (E and F). Cells treated with $100 \mu \mathrm{M}$ NSC23766 showed distinct actin cytoskeleton changes ( $\mathrm{G}$ and $\mathrm{H})$.

phalloidin and visualized the staining under a confocal microscope. As shown in Fig. 5A and B, 801D-negative control cells and $801 \mathrm{D}$ cells showed a cross-linked actin network. Deletion of Racl reduced the appearance of a cross-linked actin network and induced the reorganization of actin in 801D-shRNA cells (Fig. 5C). In addition, the formation of membrane ruffles or lamellipodia was also blocked. We further determined the roles of Rac1 in rearrangements of the actin cytoskeleton by stimulating cells with epidermal growth factor (EGF). Numerous lamellipodia and pseudopodia were produced in vector control (Fig. 5D), but not in Rac1-shRNA 801D cells (Fig. 5E and F). In order to exclude the non-specific effects of the Racl-shRNA on $801 \mathrm{D}$ cells, the distinct changes in morphology as well as the actin cytoskeletal changes in these cells following $24 \mathrm{~h}$ NSC23766 treatment was assessed (Fig. 5G and H). These results suggest that Rac1, a downstream molecule of Ras, plays a crucial role in the cytoskeleton reorganization.

The Racl-Pakl signaling pathway is involved in lung cancer cell migration and invasion. To determine the role of Pak1 in 


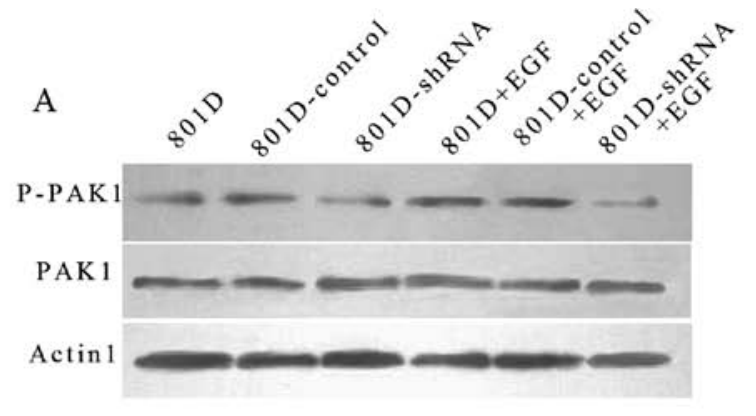

B
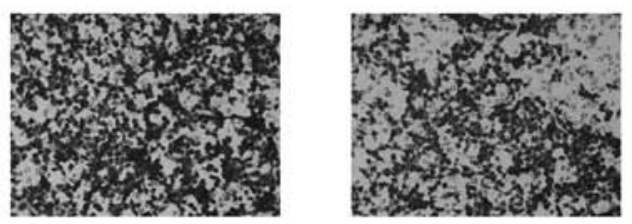

$801 \mathrm{D}+\mathrm{EGF}$

$801 \mathrm{D}+\mathrm{IPA} 3+\mathrm{EGF}$
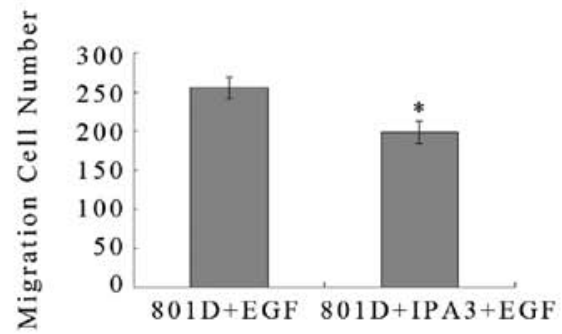

$\mathrm{C}$
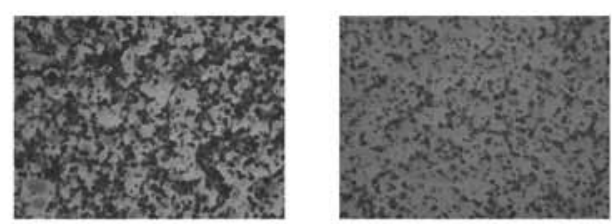

$801 \mathrm{D}+\mathrm{EGF}$

$801 \mathrm{D}+\mathrm{IPA} 3+\mathrm{EGF}$

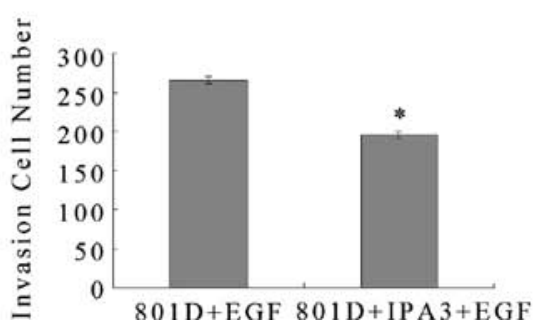

Figure 6. Role of the Pak1 pathway in Rac1 regulation of migration and invasion in lung cancer cells. Cell transfected with Rac1-shRNA or control shRNA were pretreated with EGF or without EGF, and the expression of phosphorylated and total Pak1 was examined by Western blot analysis (A). 801D cells were pre-treated with EGF or EGF and IPA3, and the migration (B) and invasion assays (C) were performed. ${ }^{*} \mathrm{P}<0.05$ compared with $801 \mathrm{D}$ cells with EGF.

Rac1-mediated lung cancer cell migration, we first examined Pak1 phosphorylation in 801D cells with $100 \mathrm{ng} / \mathrm{ml}$ EGF treatment and then tested whether inhibition of Rac1 alters Pak1 phosphorylation in response to EGF. As shown in Fig. 6A, an increase of Pak1 phosphorylation was observed following EGF treatment, indicating that EGF induces the phosphorylation of Pak1 in 801D cells. Conversely, depletion of Rac1 by shRNA transfection abolished EGF-induced phosphorylation of Pak1.
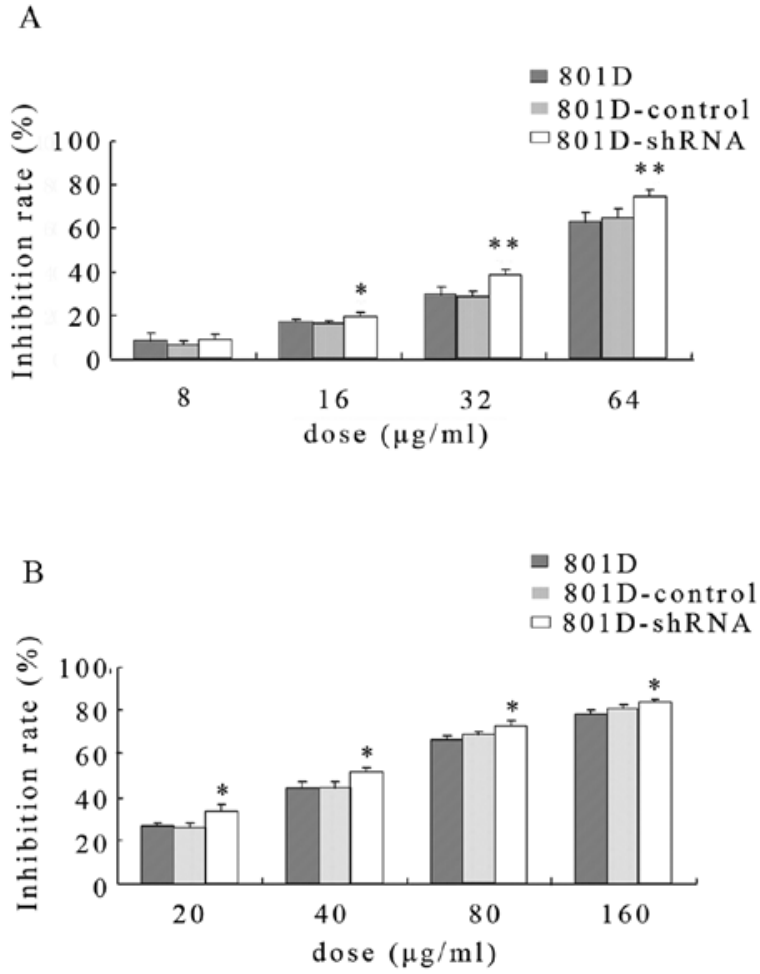

Figure 7. Effects of Rac1 specific shRNAs on antitumor drug sensitivity of $801 \mathrm{D}$ cells. $801 \mathrm{D} /$ Rac1-shRNA cells, $801 \mathrm{D} / \mathrm{Rac1}$-control cells and $801 \mathrm{D}$ cells were treated with various concentrations of the anti-tumor drugs (A) nedaplatin and (B) elemene. Cell viability was determined by the MTT assay. ${ }^{*} \mathrm{P}<0.05$ and ${ }^{* *} \mathrm{P}<0.01$ compared with control.

We then examined whether Pak1 activity correlates with lung cancer migration. To address this question, we examined whether depletion of Pak1 by IPA3, a Pak1 inhibitor, alters EGF-induced lung cancer cell migration and invasion. As shown in Fig. 6B, depletion of Pak1 levels significantly attenuated EGF-induced cell migration and invasion compared to the control cells.

Sliencing of Racl in $801 D$ cells resulted in increased chemosensitivity to elemene and nedaplatin. We next investigated whether inhibition of Rac1 by RNAi affected the sensitivity of 801D cells to the antitumor drugs, such as nedaplatin. As shown in Fig. 7A, the chemosensitivity in 801D/Rac1-siRNA cells was markedly increased at all concentration examined compared with $801 \mathrm{D}$ cells. There was no significanct difference between 801D/Rac1-control cells and 801D cells. Similar results were confirmed for the antitumor drug elemene (Fig. 7B), which indicates that this observation seems not to be drug-specific or observed by chance.

\section{Discussion}

Emerging evidence is beginning to reveal the diverse and profound role of Rac1 in regulating tumor metastasis and the tumorigenic process, raising the possibilities that Rac1 may be a useful prognostic indicator $(4,5,7,8)$. Expression of Rac1 protein has been reported to be greater in testicular germ cell tumors of higher stages than of lower stages $(16,17)$. It has also been shown that the increased expression of Rac1 is related 
to higher TNM stages of gastric carcinoma $(18,19)$. These findings suggest the role of Racl overexpression in human tumors and the correlation of Racl overexpression with a malignant phenotype. In the current study, we examined the expression of Rac1 in patients with non-small cell lung cancer (NSCLC) and compared it to clinicopathological parameters by immunohistochemistry. We found that Rac1 expression was correlated with lymphatic metastasis and TNM stages, while no association was observed between Rac1 expression and any other clinicopathologic parameters. Additionally, Kaplan-Meier analysis revealed a significant association of Rac1 expression with 5-year survival rates. These observations are well-correlated with the recent studies demonstrating that overexpression of Racl plays a crucial role in progression and metastasis of lung cancer cells $(5,20)$.

Different studies have indicated contradictory roles for Rac1 in tumor progression. For example, although in most human malignancies Rac1 down-regulation was shown to result in inhibition of migration $(17,19,22,23)$, migration was shown to be stimulated in the aggressive breast cancer cells where Rac1 was knocked down $(21,24)$. These issues indicate a complex network of Rac1 in the tumor process that results in a particular biological outcome in each tumor type. In our experiments, we found that Rac1 expression positively associated with the metastatic potential of human lung cancer cells. Knocking down of Rac1 expression by shRNA against Rac1 suppressed lung cancer cell migration and invasion. Similarly, NSC23766, an inhibitor of Rac1 activity, could inhibit lung cancer cell migration and invasion. Our results indicate that Racl plays a key role in the vigorous migration and invasion of lung cancer cells.

The Rho GTPases are reported to be key regulators of actin dynamics that lead to organized actin-based structures associated with cell migration. Activated Rac1 stimulates lamellipodia formation (22). In the present study, we reported the roles of Rac1 in the organization of the actin cytoskeleton and cell migration in $801 \mathrm{D}$ cells. Our results showed that shRNA-mediated deletion of Rac1 resulted in fewer crosslinked actin networks and strongly inhibited actin-positive membrane ruffles, lamellipodia, and pseudopodia formation. Conversely, expression of the Rac1 protein in control 801D cells led to the formation of lamellipodia. Treatment with a Rac1 inhibitor resulted in distinct changes in morphology as well as actin cytoskeletal changes in these cells, which is very similar to the effects of Rac1-shRNA. In addition, we also determined the roles of Racl in the rearrangements of the actin cytoskeleton by stimulating cells with EGF. EGF increased lamellipodia and pseudopodia formation in vector control, but not in Rac1-shRNA 801D cells. These results are consistent with the reported observations that depletion of Rac1 in colorectal carcinoma cells strongly inhibited rearrangements of the actin cytoskeleton $(14,18)$. Consistent with our findings, the activation of Racl is required for the EGF-induced morphological changes by regulating the organization of the actin cytoskeleton (23).

However, the mechanism by which Racl increases the invasive potential remains unclear. Racl and Pak1 have recently been shown to be key regulators of cancer cell signaling networks, and there are several lines of evidence linking Racl and Pak1 to the acquisition of a migratory, invasive, and metastatic phenotype and to a variety of processes that occur in tumors, including cell transformation, survival, invasion, metastasis, and angiogenesis $(9,10,12,13)$. Pak1 binds to Rac1 in a GTP-dependent manner, after which activated Pak1 regulates cellular functions such as cytoskeleton dynamics, cell adhesion, and transcription $(10,12)$. Furthermore, several growth factors including the epidermal growth factor (EGF) could activate Pak1 and subsequently promote tumor cell invasion (24). To address this question, we examined Pak1 phosphorylation levels in 801D cells after treatment with $100 \mathrm{ng} / \mathrm{ml}$ EGF, and found an increase of Pak1 phosphorylation. Conversely, depletion of Rac1 by shRNA transfection abolished EGF-induced phosphorylation of Pak1. Additionally, IPA3, a Pak1 inhibitor, could alter EGF-induced lung cancer cell migration and invasion, which indicates that the Rac1-Pak1 pathway may be a potential therapeutic target for the prevention of tumor invasion and metastasis by inhibition of this signaling pathway.

Nedaplatin has been developed as a second-generation platinum complex with pronounced clinical antitumor activity against solid tumors but with lower nephrotoxicity than cisplatin (25). Elemene (1-methyl-1-vinyl-2, 4-diisopropenylcyclohexane) is a novel anticancer drug extracted from the traditional Chinese medicinal herb Rhizoma zedoariae. It has been effectively used in China to treat certain types of tumors (26), which displayed low toxicity with no observable liver, kidney, or bone marrow toxicities. Therefore, for this investigation, we used these two chemotherapeutic drugs. The MTT results revealed that down-regulation of Rac1 also increased chemosensitivity to the antitumor drugs elemene and nedaplatin in $801 \mathrm{D}$ cells. To our knowledge, this is the first report describing a relationship between a cytoskeletalrelated protein and the sensitivity to elemene and nedaplatin, which are widely used in the treatment of solid tumors. This observation raised the possibility that the level of expression of Rac1 in tumor samples could be correlated with sensitivity to elemene and nedaplatin in the clinic. Studies of Rac1 in samples of elemene and nedaplatin-treated patients are thus warranted.

In summary, our data demonstrate that suppression of Racl could decrease the invasive potential of lung cancer and induce the rearrangements of actin cytoskeleton in vitro. Pak1, a major common downstream effector for Rac1, also could play a critical role in Rac1-mediated invasion and metastasis. Furthermore, Rac1 was found to be overexpressed in tumor tissues, and its expression was directly correlated with tumor stage, lymph node metastasis, and patient survival. We also showed that down-regulation of Racl enhanced lung cancer cell sensitivity to the chemotherapeutic drugs elemene and nedaplatin. Our findings indicate that Rac1 may be a potential target for therapeutic anti-cancer drugs and we provide new insight into the development of gene therapy technology to treat patients with lung cancer.

\section{Acknowledgements}

This project was supported by the Hangzhou Medicine Science Research Fund (Project, no. 2009B088) and the Zhejiang Chinese Medical Science Research Project (no. 2008A144). We would like to thank Dr Kedi Xu and Dr Chunlei Fan for their technical assistance and discussion. 


\section{References}

1. Heasman SJ and Ridley AJ: Mammalian Rho GTPases: new insights into their functions from in vivo studies. Nat Rev Mol Cell Biol 9: 690-701, 2008.

2. Price LS and Collard JG: Regulation of the cytoskeleton by Rho-family GTPases: implications for tumour cell invasion. Semin Cancer Biol 11: 167-173, 2001.

3. Schmitz AA, Govek EE, Bottner B and Van Aelst L: Rho GTPases: signaling, migration, and invasion. Exp Cell Res 261: 1-12, 2000.

4. Gomez del Pulgar T, Benitah SA, Valeron PF, Espina C and Lacal JC: Rho GTPase expression in tumourigenesis: evidence for a significant link. Bioessays 27: 602-613, 2005.

5. Yuan K, Qian C and Zheng R: Prognostic significance of immunohistochemical Rac1 expression in survival in early operable non-small cell lung cancer. Med Sci Monit 15: BR313-BR319, 2009.

6. Espina C, Cespedes MV, Garcia-Cabezas MA, et al: A critical role for Rac1 in tumor progression of human colorectal adenocarcinoma cells. Am J Pathol 172: 156-166, 2008.

7. Chan AY, Coniglio SJ, Chuang YY, et al: Roles of the Rac1 and Rac3 GTPases in human tumor cell invasion. Oncogene 24 7821-7829, 2005.

8. Niggli V, Schlicht D and Affentranger S: Specific roles of Rac1 and Rac2 in motile functions of HT1080 fibrosarcoma cells. Biochem Biophys Res Commun 386: 688-692, 2009.

9. Kumar R, Gururaj AE and Barnes CJ: p21-activated kinases in cancer. Nat Rev Cancer 6: 459-471, 2006.

10. Balasenthil S, Sahin AA, Barnes CJ, et al: p21-activated kinase-1 signaling mediates cyclin D1 expression in mammary epithelial and cancer cells. J Biol Chem 279: 1422-1428, 2004.

11. Travis WD, Brambilla E, Muller-Hermelink HK and Harris CC: Pathology and Genetics of Tumours of the Lung, Pleura, Thymus and Heart. World Health Organization. International Agency for Research on Cancer. IARC Press, Lyon, 2004.

12. Watanabe Y: TNM classification for lung cancer. Ann Thorac Cardiovasc Surg 9: 343-350, 2003.

13. Walch A, Seidl S, Hermannstadter C, et al: Combined analysis of Rac1, IQGAP1, Tiam1 and E-cadherin expression in gastric cancer. Mod Pathol 21: 544-552, 2008.

14. Xie JJ, Xu LY, Xie YM, et al: Involvement of Cyr61 in the growth, invasiveness and adhesion of esophageal squamous cell carcinoma cells. Int J Mol Med 27: 429-434, 2011.
15. Chen Q, Wang Y, Xu K, et al: Curcumin induces apoptosis in human lung adenocarcinoma A549 cells through a reactive oxygen species-dependent mitochondrial signaling pathway. Oncol Rep 23: 397-403, 2010.

16. Kamai T, Yamanishi T, Shirataki H, et al: Overexpression of RhoA, Rac1, and Cdc42 GTPases is associated with progression in testicular cancer. Clin Cancer Res 10: 4799-4805, 2004.

17. Ridley AJ: Rho GTPases and cell migration. J Cell Sci 114: 2713-2722, 2001

18. Pan Y, Bi F, Liu N, et al: Expression of seven main Rho family members in gastric carcinoma. Biochem Biophys Res Commun 315: 686-691, 2004.

19. Kawasaki Y, Sato R and Akiyama T: Mutated APC and Asef are involved in the migration of colorectal tumour cells. Nat Cell Biol 5: 211-215, 2003.

20. Liu Y, Wang Y, Zhang Y, et al: Abnormal expression of p120catenin, E-cadherin, and small GTPases is significantly associated with malignant phenotype of human lung cancer. Lung Cancer 63: 375-382, 2009.

21. Zuo Y, Shields SK and Chakraborty C: Enhanced intrinsic migration of aggressive breast cancer cells by inhibition of Racl GTPase. Biochem Biophys Res Commun 351: 361-367, 2006.

22. Raftopoulou M and Hall A: Cell migration: Rho GTPases lead the way. Dev Biol 265: 23-32, 2004.

23. Kurokawa K, Itoh RE, Yoshizaki H, Nakamura YO and Matsuda M: Coactivation of Rac1 and $\mathrm{Cdc} 42$ at lamellipodia and membrane ruffles induced by epidermal growth factor. Mol Biol Cell 15: 1003-1010, 2004.

24. He H, Levitzki A, Zhu HJ, Walker F, Burgess A and Maruta H: Platelet-derived growth factor requires epidermal growth factor receptor to activate p21-activated kinase family kinases. J Biol Chem 276: 26741-26744, 2001.

25. Masago K,Fujita S, Kim YH, et al: Phase I study of the combination of nedaplatin and gemcitabine in previously untreated advanced squamous cell lung cancer. Cancer Chemother Pharmacol 67: 325-330, 2011.

26. Li QQ, Wang G, Zhang M, Cuff CF, Huang L and Reed E: $\beta$-elemene, a novel plant-derived antineoplastic agent, increases cisplatin chemosensitivity of lung tumor cells by triggering apoptosis. Oncol Rep 22: 161-170, 2009. 